\title{
Valores de referencia para parámetros determinantes del estado de equilibrio ácido-base en mulas sanas a gran altura
}

\section{Reference values for acid-base parameters in healthy mules at high altitude}

\author{
Johanna Patricia Vanegas Rincón ${ }^{1}$, Pedro Vargas-Pinto², \\ Claudia Valderrama Martínez ${ }^{2,3}$
}

\section{Resumen}

Se estimaron intervalos de referencia (IR) de parámetros fisiológicos relacionados con el estado de equilibrio acido-base a partir de muestras de sangre de 76 de mulas sanas a gran altura. Los animales estuvieron en reposo y sometidos a una misma dieta. Los datos obtenidos fueron comparados con los reportados por varios autores, encontrando cercanía entre los IR estimados y los de los autores citados. Se concluyó que los IR de mulas y caballos no presentan grandes diferencias.

Palabras clave: intervalos de referencia, equilibrio ácido-base, $\mathrm{pH}$, mulas

\section{AbSTRACT}

Reference intervals (RI) of physiological parameters related to the acid-base equilibrium state were estimated from blood samples of 76 healthy mules at high altitude. The animals were at rest and subjected to the same diet. The data obtained were compared with those reported by various authors, finding closeness between the estimated RIs and those of the cited authors. It was concluded that the RI of mules and horses do not present great differences.

Key words: reference intervals, acid-base balance, $\mathrm{pH}$, mules

\footnotetext{
${ }^{1}$ Facultad de Ciencias Agropecuarias, Universidad De La Salle, Bogotá, Colombia

${ }^{2}$ Grupo de Investigación en Ciencias Biomédicas y Veterinarias, Facultad de Ciencias Agropecuarias, Universidad De La Salle, Bogotá, Colombia

${ }^{3}$ E-mail: ccvalderrama@unisalle.edu.co
} 


\section{INTRODUCCIÓN}

La mula es un animal de trabajo importante para algunos sistemas productivos en el mundo, debido a su adaptabilidad, fuerza y resistencia, bajos requerimientos nutricionales y poco consumo de agua. En Colombia, aunque es ampliamente utilizada como animal de carga, puesto que sus características son ideales en regiones montañosas de difícil acceso (González, 2015), también se emplean como animales de equitación para el trabajo policial. La mula (Equus mulus) es un équido híbrido proveniente del cruzamiento entre un burro (Equus asinus) y una yegua (Equus caballus), el cual presenta características fenotípicas similares a la de sus progenitores; sin embargo, posee una resistencia y fuerza superior a estos. Pese a la importancia que tiene la mula, los datos fisiológicos reportados para esta especie son escasos (Herrera, 2018), lo cual conlleva a que los médicos veterinarios utilicen valores de referencia de caballos en situaciones clínicas, que pueden conducir a diagnósticos erróneos (Soma et al., 1996; McLean et al., 2016).

En medicina veterinaria, la estandarización de parámetros fisiológicos es importante para la identificación del estado clínico de una población, ya que facilita el diagnóstico temprano de procesos patológicos infecciosos, minimizando la morbilidad y la mortalidad. De este modo, la alteración del equilibrio acido-base es de vital importancia para el diagnóstico clínico. Este es explicado mediante dos modelos, el enfoque tradicional de $\mathrm{H}-\mathrm{H}$, Henderson-Hasselbalch, que permite establecer el estado ácido-base, y el enfoque relativamente nuevo de Stewart, que propone mecanismos iónicos como la explicación de dicha alteración. De manera clásica se utiliza la ecuación Henderson-Hasselbalch para identificar problemas respiratorios $\left(\mathrm{CO}_{2}\right.$ anormal) o metabólicos ( $\mathrm{HCO}_{3}{ }^{-}$anormal), así como el exceso de base (EB) para valorar la magnitud de la anormalidad metabólica y la brecha aniónica (AG) que se calcula para detectar aniones no identificados en plasma (Rodríguez, 2015).
El modelo de Stewart tiene un enfoque fisicoquímico cuantitativo, relacionado a modificaciones en tres variables dependientes que son: la diferencia de iones fuertes (SID), los ácidos débiles totales $\left(\mathrm{A}_{\text {тот }}\right)$ y la presión parcial de dióxido de carbono $\left(\mathrm{pCO}_{2}\right)$. Dado el enfoque mecanicista de este modelo, es clínicamente recomendado siempre que las concentraciones séricas totales de proteína, albúmina o fosfato sean notablemente anormales (Constable, 2000).

Debido a los vacíos encontrados en la literatura, respecto a la información acerca de los parámetros fisiológicos diagnósticos para mulas, en este trabajo se plantea como objetivo establecer intervalos de referencia para parámetros fisiológicos de mulas sanas a gran altitud, que permitan establecer el estado ácido-base.

\section{Materiales y Métodos}

El estudio fue aprobado por el Comité de Ética para Pruebas en Animales de la Universidad de la Salle (Aprobación \# 0047, 31 de mayo de 2019). Se seleccionaron 76 mulas sanas de propiedad de la Policía Nacional de Colombia, que tuvieron acceso a pastos, agua y fueron alimentados con raciones (provistos de dietas de cereales). Ninguno de los animales tenía antecedentes de enfermedad aguda o crónica en los últimos cuatro meses. El muestreo se realizó en el municipio Santa Rosa de Viterbo, a una altitud de $2753 \mathrm{~m}$ y temperatura media de $16^{\circ} \mathrm{C}$, y en el distrito capital de Bogotá (DC) a 2640 msnm y temperatura promedio de $15{ }^{\circ} \mathrm{C}$.

Los especímenes tuvieron un tiempo de reposo de un mínimo de cinco días antes de la prueba. Previo a la prueba, un veterinario experimentado realizó un examen clínico completo a los animales, Los datos clínicos registrados incluyeron sexo, edad, actitud, temperamento, membranas mucosas, tiempo de llenado capilar, frecuencia cardíaca y respiratoria, motilidad intestinal (evaluada por aus- 
cultación de los cuatro cuadrantes abdominales) y temperatura rectal (usando un termómetro de mercurio). La prueba fue realizada entre las 07:00 y 10:00 y las 14:00 y 16:00 para evitar altas temperaturas que pudiera afectar los resultados del estudio.

Se tomaron muestras de sangre $(5 \mathrm{ml})$ de sangre por punción de la vena yugular. En forma inmediata se procesaron $0.5 \mathrm{ml}$ de la muestra en un dispositivo de punto de atención (sistema de análisis de gases en sangre epoc ${ }^{\circledR}$ HR-1002-00-00, Siemens Healthcare) para obtener las siguientes determinaciones: $\mathrm{pH}$, presión de dióxido de carbono (pCO2), presión de oxígeno $\left(\mathrm{pO}_{2}\right)$, ion bicarbonato $\left(\mathrm{HCO}_{3}^{-}\right)$, exceso de base (EB), tensión de dióxido de carbono $\left(\mathrm{tCO}_{2}\right)$, sodio $\left(\mathrm{Na}^{+}\right)$, cloruro $\left(\mathrm{Cl}^{-}\right)$, potasio $\left(\mathrm{K}^{+}\right)$, calcio ionizado $\left(\mathrm{Ca}^{++}\right)$, brecha aniónica $(\mathrm{AG})$, volumen celular empaquetado (PCV), glucosa, creatinina y lactato. La muestra de sangre restante (4.5 $\mathrm{ml})$ se colocó en un tubo «Vacutainer» con EDTA, se almacenó a $6{ }^{\circ} \mathrm{C}$ y se transportó al laboratorio (Laboratorio Clínico, Clínica Veterinaria, Universidad de La Salle) para obtener el valor total de proteína plasmática, por medio de un refractómetro óptico.

La diferencia de iones fuertes (SID) y $\left(\mathrm{A}_{\text {тот }}\right)$ concentración total de aniones de tampón no volátiles se calcularon utilizando el software Stewart Acid-Base Analysis (Equine) disponible en: https://ovc.uoguelph.ca/doc/henry-staempfli-acid-baseanalysis/2-0.html.

La normalidad de los datos fue verificada mediante el test Shapiro-Wilk. A los datos no normales se les aplicó la transformación Box-Cox seguido de la prueba indicada. Los intervalos de referencia (IR) fueron estimados usando el método paramétrico, de acuerdo con el tamaño muestral (Ichihara y Boyd, 2010; Friedrichs et al., 2012) Para la depuración de los outliers se aplicó el método de Tukey. Todas las variables fueron procesadas y analizadas por medio del software R-Proyect en el entorno R-studio. El valor de $p$ utilizado fue de $p<0.05$.

\section{Resultados}

Se determinaron los IR para 18 parámetros fisiológicos determinantes en el estado de equilibrio ácido-base y relacionadas mediante las ecuaciones desarrolladas por $\mathrm{H}-\mathrm{H}$ y Stewart, en mulas a gran altura (Cuadro 1).

En el presente trabajo se hallaron los IR de los parámetros de equilibrio acido-base para mulas a partir de 76 ejemplares sanos y se compararon con los establecidos para caballos (Soma et al., 1996; Navarro et al., 2005; Viu et al., 2010; Gómez Nieto, 2011), esperando hallar similitudes (Cuadro 2), ya que son especies pertenecientes al mismo género. No fue posible realizar un análisis de partición en subclases, como sexo o edad, ya que sólo se contaba con 36 ejemplares hembras y 40 machos; además, el número de observaciones en 11 de las variables se vio reducido por la eliminación de valores atípicos. Se hace notar que este análisis debe realizarse por lo menos a partir de 40 observaciones por clase (Friedrichs et al., 2012).

Adicionalmente se planteó un criterio de comparación definido por la razón entre los límites de los IR y que denota el coeficiente de límite (CL). Dicha razón puede ser mayor, menor o igual a uno. $\mathrm{Si} C L=1$ implica que los limites son exactamente iguales, si CL $>1$ implica que el límite estimado es mayor que de los demás autores, si $\mathrm{CL}<1$ implica que el límite hallado es menor que el de los demás autores.

En el equilibrio del estado ácido-base, el ion hidrógeno $(\mathrm{H}+)$ es uno de los parámetros de mayor importancia y de acuerdo con lo descrito por H-H en su ecuación, este depende de la interacción entre $\mathrm{pCO}_{2} \mathrm{y}$ la concentración en plasma $\mathrm{cHCO}_{3}$ (Hasselbalch, 1917). De acuerdo con esto, al comparar los IR de $\mathrm{pCO} 2$ para mulas con los de caballos, estos se encuentran dentro los parámetros establecidos por otros autores (Cuadro 2). Sin embargo, el CL muestra que los valores para mulas están más cerca de 
Cuadro 1. Intervalos de referencia para las variables determinantes del estado ácido base en mulas

\begin{tabular}{lcc}
\hline Variable & Media $\pm \mathrm{DE}$ & Intervalo de Referencia \\
\hline $\mathrm{pH}$ & $7.417 \pm 0.023$ & $7.36-7.45$ \\
$\mathrm{pCO} 2 \mathrm{mmHg}$ & $41.15 \pm 2.963$ & $35.3-46.9$ \\
$\mathrm{pO} 2 \mathrm{mmHg}$ & $34.60 \pm 4.084$ & $28.16-40.88$ \\
$\mathrm{cHCO} 3 \mathrm{mmol} / \mathrm{l}$ & $26.34 \pm 2.316$ & $22-30.8$ \\
$\mathrm{Na}+\mathrm{mmol} / \mathrm{l}$ & $138.9 \pm 1.548$ & $136.1-142$ \\
$\mathrm{~K}+\mathrm{mmol} / \mathrm{l}$ & $4.1 \pm 0.362$ & $3.39-4.7$ \\
$\mathrm{Ca}++\mathrm{mmol} / \mathrm{l}$ & $1.728 \pm 0.103$ & $1.4-1.9$ \\
$\mathrm{Cl}-\mathrm{mmol} / \mathrm{l}$ & $102.2 \pm 2.651$ & $96.3-106$ \\
$\mathrm{cTCO} 2 \mathrm{mmol} / \mathrm{l}$ & $27.78 \pm 2.953$ & $23.8-32.8$ \\
$\mathrm{aGap} \mathrm{mmol} / \mathrm{l}$ & $10.34 \pm 1.367$ & $8-12.5$ \\
$\mathrm{Hto} \%$ & $40.90 \pm 4.999$ & $31.5-74.5$ \\
$\mathrm{Glu} \mathrm{mg} / \mathrm{dl}$ & $93.85 \pm 14.14$ & $71.3-121.9$ \\
Lac mmol/1 & $0.918 \pm 0.502$ & $0.39-2.22$ \\
$\mathrm{Crea} \mathrm{mg} / \mathrm{dl}$ & $1.528 \pm 0.262$ & $1-2$ \\
$\mathrm{SID}$ & $39.72 \pm 2.403$ & $35-43.5$ \\
A & $12.35 \pm 0.752$ & $10.9-13.8$ \\
$\mathrm{TPP} \mathrm{g} / \mathrm{l}$ & $69.31 \pm 4.376$ & $61.7-78.9$ \\
$\mathrm{BE}(\mathrm{mEq} / \mathrm{l})$ & $2.047 \pm 2.352$ & $-2.6-6.65$ \\
\hline
\end{tabular}

pCO2: presión de dióxido de carbono; $\mathrm{pO} 2$ : presión de oxígeno, $\mathrm{CHCO}_{3}$; bicarbonato calculado; (pO2): presión de oxígeno, $\mathrm{Na}+$ : sodio; $\mathrm{Cl}$-: cloruro; $\mathrm{K}+$; potasio; $\mathrm{Ca}++$; calcio ionizado; tCO2: tensión de dióxido de carbono aGap: Anion Gap, Hto: hematocrito; Glu: glucosa; Lac: lactato; Crea: creatinina; SID: Diferencia de iones fuertes; Атот: ácidos débiles totales; TPP: proteínas plasmáticas totales; $\mathrm{BE}$ : exceso de base

los reportados por Navarro et al. (2005), seguido por los reportados por Gómez Nieto (2011), distando en un pequeño porcentaje de los de Viu et al., (2010) y Soma et al. (1996). En cuanto a los parámetros calculados para el $\mathrm{CHCO}_{3}^{-}$de mulas, el CL muestra gran cercanía con los de caballos, existiendo una pequeña diferencia con lo reportado por Soma et al. (1996) en el límite inferior del intervalo.

Con el objetivo de complementar la aproximación clásica, fueron descritos dos nuevos modelos matemáticos, el cálculo del
EB y AG (Andersen et al., 1960). El EB es una herramienta práctica que permite calcular el déficit de ácido o base para el estado de equilibrio y la concentración de $\mathrm{HCO}_{3}$ requerida para corregir dicha deficiencia, lo cual permite valorar la magnitud de la anormalidad metabólica (Gómez Nieto, 2011). Como se puede observar a partir de los CL en la comparación entre mulas y caballos, el límite inferior de IR para EB de los primeros dista considerablemente de los descritos para los segundos, siendo el límite superior más congruente entre las dos especies. Sin em- 
Cuadro 2. Comparación de Intervalos de referencia (IR) de parámetros físiológicos del estado de equilibrio acido-base entre caballos y mulas

\begin{tabular}{|c|c|c|c|c|c|c|c|c|c|}
\hline \multirow{3}{*}{ Variable } & \multirow{3}{*}{ 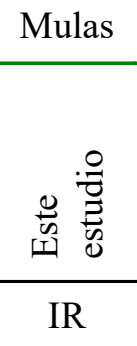 } & \multicolumn{8}{|c|}{ Caballos } \\
\hline & & \multicolumn{2}{|c|}{ 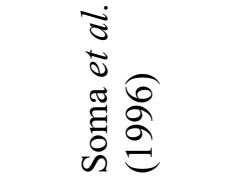 } & \multicolumn{2}{|c|}{ 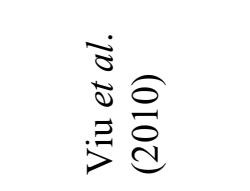 } & \multicolumn{2}{|c|}{ 总 } & \multicolumn{2}{|c|}{ 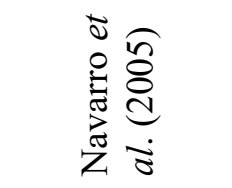 } \\
\hline & & $\mathrm{IR}^{1}$ & CL & $\mathrm{IR}^{2}$ & $\mathrm{CL}$ & $\mathrm{IR}^{3}$ & CL & $\mathrm{IR}^{4}$ & $\mathrm{CL}$ \\
\hline $\mathrm{pH}$ & $\begin{array}{l}7.36- \\
7.45\end{array}$ & $\begin{array}{l}7.3- \\
7.4\end{array}$ & $\begin{array}{l}1.008 / \\
1.006\end{array}$ & $\begin{array}{l}7.31- \\
7.45\end{array}$ & $\begin{array}{c}1.006 / \\
1\end{array}$ & $\begin{array}{l}7.33- \\
7.45\end{array}$ & $\begin{array}{c}1.004 / \\
1\end{array}$ & $\begin{array}{l}7.31- \\
7.45\end{array}$ & $\begin{array}{c}1.006 / \\
1\end{array}$ \\
\hline $\mathrm{pCO}_{2} \mathrm{mmHg}$ & $\begin{array}{c}35.3- \\
46.9\end{array}$ & $\begin{array}{c}45.7- \\
55.7\end{array}$ & $\begin{array}{l}0.772 / \\
0.842\end{array}$ & $\begin{array}{c}41- \\
53\end{array}$ & $\begin{array}{c}0.860 / \\
0.884\end{array}$ & $\begin{array}{c}34- \\
53\end{array}$ & $\begin{array}{l}1.038 / \\
0.884\end{array}$ & $\begin{array}{c}38- \\
45\end{array}$ & $\begin{array}{l}0.928 / \\
1.042\end{array}$ \\
\hline $\mathrm{pO}_{2} \mathrm{mmHg}$ & $\begin{array}{c}28.16- \\
40.88\end{array}$ & & & & & & & & \\
\hline $\mathrm{cHCO}_{3} \mathrm{mmol} / \mathrm{L}$ & $\begin{array}{c}22- \\
30.8\end{array}$ & $\begin{array}{c}24.6- \\
33.6\end{array}$ & $\begin{array}{c}0.894 / \\
0.916\end{array}$ & $\begin{array}{c}24- \\
30\end{array}$ & $\begin{array}{c}0.916 / \\
1.026\end{array}$ & $\begin{array}{c}24- \\
31\end{array}$ & $\begin{array}{c}0.916 / \\
0.993\end{array}$ & $\begin{array}{c}24- \\
30\end{array}$ & $\begin{array}{c}0.916 / \\
1.026\end{array}$ \\
\hline $\mathrm{Na}+\mathrm{mEq} / 1$ & $\begin{array}{c}136.1- \\
142\end{array}$ & & & $\begin{array}{c}134- \\
144\end{array}$ & $\begin{array}{l}1.015 / \\
0.986\end{array}$ & $\begin{array}{c}132- \\
142\end{array}$ & $\begin{array}{c}1.031 \\
/ 1\end{array}$ & $\begin{array}{c}134- \\
144\end{array}$ & $\begin{array}{c}1.015 / \\
0.986\end{array}$ \\
\hline $\mathrm{K}+\mathrm{mEq} / 1$ & $\begin{array}{c}3.39- \\
4.7\end{array}$ & & & $\begin{array}{c}3.5- \\
4.5\end{array}$ & $\begin{array}{c}0.968 / \\
1.044\end{array}$ & $\begin{array}{c}2.4- \\
4.6\end{array}$ & $\begin{array}{l}1.412 / \\
1.021\end{array}$ & $\begin{array}{l}3.5- \\
4.5\end{array}$ & $\begin{array}{c}0.968 / \\
1.044\end{array}$ \\
\hline $\mathrm{Ca}++\mathrm{mmol} / \mathrm{l}$ & $\begin{array}{c}1.4- \\
1.9\end{array}$ & & & $\begin{array}{l}1.4- \\
1.6\end{array}$ & $\begin{array}{c}1 / \\
1.187\end{array}$ & $\begin{array}{c}1.26- \\
1.9\end{array}$ & $\begin{array}{c}1.111 / \\
1\end{array}$ & $\begin{array}{l}1.4- \\
1.6\end{array}$ & $\begin{array}{c}1 / \\
1.187\end{array}$ \\
\hline Cl-mEq/1 & $\begin{array}{c}96.3- \\
106\end{array}$ & & & $\begin{array}{l}90- \\
100\end{array}$ & $\begin{array}{l}1.07 / \\
1.06\end{array}$ & $\begin{array}{l}97- \\
105\end{array}$ & $\begin{array}{c}0.992 / \\
1.009\end{array}$ & $\begin{array}{l}90- \\
100\end{array}$ & $\begin{array}{l}1.07 / \\
1.06\end{array}$ \\
\hline $\mathrm{cTCO}_{2} \mathrm{mmHg}$ & $\begin{array}{c}23.8- \\
32.8\end{array}$ & & & $\begin{array}{c}28- \\
35\end{array}$ & $\begin{array}{l}0.85 / \\
0.937\end{array}$ & & & $\begin{array}{c}28- \\
35\end{array}$ & $\begin{array}{l}0.85 / \\
0.937\end{array}$ \\
\hline $\mathrm{AG} \mathrm{mmol/1}$ & $\begin{array}{c}8 \\
12.5\end{array}$ & & & & & $\begin{array}{l}12- \\
18\end{array}$ & $\begin{array}{c}0.666 / \\
0.694\end{array}$ & $\begin{array}{l}7- \\
15\end{array}$ & $\begin{array}{l}1.142 / \\
0.833\end{array}$ \\
\hline PCV\% & $\begin{array}{l}31.5- \\
74.5\end{array}$ & & & $\begin{array}{c}36- \\
44\end{array}$ & $\begin{array}{c}0.875 / \\
1.693\end{array}$ & & & & \\
\hline Glu (mg/dl) & $\begin{array}{l}71.3- \\
121.9\end{array}$ & & & $\begin{array}{l}98.1- \\
107.7\end{array}$ & $\begin{array}{l}13.08 / \\
20.66\end{array}$ & & & & \\
\hline $\mathrm{Lac}(\mathrm{mmol} / \mathrm{L})$ & $\begin{array}{l}0.39- \\
2.22\end{array}$ & & & $<2$ & $\begin{array}{l}--/ \\
1.1\end{array}$ & $\begin{array}{l}0- \\
2\end{array}$ & $--/ 1.11$ & & \\
\hline Crea $(\mathrm{mg} / \mathrm{dl})$ & $\begin{array}{l}1- \\
2\end{array}$ & & & $<20$ & & & & & \\
\hline SID & $\begin{array}{c}35- \\
43.5\end{array}$ & & & $\begin{array}{c}35- \\
38.5\end{array}$ & $\begin{array}{c}1 / \\
1.129\end{array}$ & $\begin{array}{c}38- \\
44\end{array}$ & $\begin{array}{c}0.921 / \\
0.988\end{array}$ & $\begin{array}{c}38- \\
44\end{array}$ & $\begin{array}{c}0.921 / \\
0.988\end{array}$ \\
\hline $\mathrm{A}_{\mathrm{TOT}}$ & $\begin{array}{l}10.9- \\
13.8\end{array}$ & & & $\begin{array}{c}12- \\
15\end{array}$ & $\begin{array}{c}0.908 / \\
0.92\end{array}$ & $\begin{array}{l}12- \\
16\end{array}$ & $\begin{array}{c}0.908 / \\
0.862\end{array}$ & $\begin{array}{c}14- \\
15.6\end{array}$ & $\begin{array}{c}0.778 / \\
0.884\end{array}$ \\
\hline TPP (g/L) & $\begin{array}{l}61.7- \\
78.9\end{array}$ & & & $\begin{array}{l}65- \\
75\end{array}$ & $\begin{array}{l}0.949 / \\
1.052\end{array}$ & $\begin{array}{l}54- \\
75\end{array}$ & $\begin{array}{l}1.142 / \\
1.052\end{array}$ & $\begin{array}{l}65- \\
75\end{array}$ & $\begin{array}{c}0.949 / \\
1.052\end{array}$ \\
\hline EB (mEq/l) & $\begin{array}{l}-2.6- \\
6.65\end{array}$ & $\begin{array}{c}-0.9- \\
7\end{array}$ & $\begin{array}{c}2.888 / \\
0.95\end{array}$ & $\begin{array}{c}-6- \\
6\end{array}$ & $\begin{array}{c}0.433 / \\
0.108\end{array}$ & $\begin{array}{c}-1- \\
5\end{array}$ & $\begin{array}{l}2.6 / \\
1.33\end{array}$ & $\begin{array}{c}-6- \\
6\end{array}$ & $\begin{array}{c}0.433 / \\
1.108\end{array}$ \\
\hline
\end{tabular}


bargo, cabe notar que los valores del límite inferior varían entre todos los rangos reportados y no solos en los hallados para mulas.

El AG se utiliza para el diagnóstico de la acidosis metabólica y representa la diferencia entre las concentraciones de aniones y cationes no medibles (Constable, 2000). Aunque este parámetro no se encuentra reportado en el trabajo de Soma et al. (1996) ni en el de Viu et al. (2010), muestra cercanía con lo reportado por Navarro et al. (2005), distando considerablemente de los reportados por Gómez Nieto (2011).

$\mathrm{SID} \mathrm{y}_{\text {тот }}$, al igual que $\mathrm{pCO} 2$, son utilizados en el modelo cuantitativo de análisis fisicoquímico del estado acido-base en soluciones acuosas de Stewart (1983). Según este modelo, diferentes variables dependientes e independientes interactúan afectando la concentración de $\mathrm{H}^{+}$en los distintos fluidos corporales, donde las variables independientes propuestas son: la $\mathrm{pCO}_{2}$, $\mathrm{SID}$ y $\mathrm{AG}\left(\mathrm{A}_{\mathrm{TOT}}\right)$ (Martínez y Oliver, 2016). Según esto, la SID corresponde a la diferencia entre la sumatoria de la concentración de los cationes fuertes y la concentración de los aniones fuertes; es decir, a la carga neta de los electrolitos fuertes (Stewart, 1983). Cuando se compara los IR calculados para mulas con los de caballos, los CL permiten evidenciar que los datos son relativamente cercanos a los reportados por Viu et al. (2010), Navarro et al. (2005) y Gómez Nieto (2011).

$\mathrm{A}_{\text {тот }}$ representa la concentración total de ácidos volátiles débiles presentes en el plasma, donde los mayores representantes son los fosfatos y las proteínas séricas, especialmente la albúmina. Un cambio en la concentración de dichos ácidos altera las concentraciones de los iones hidrogeno. A partir de la interpretación de los CL, los IR de mulas para este parámetro muestra cercanía con los reportados para Viu et al. (2010) y Gómez Nieto (2011), observando una pequeña diferencia con lo reportado por Navarro et al. (2005); sin embargo, se puede decir que los rangos para mulas no distan de lo reportado para caballos.
Los IR hallados para mulas de los electrolitos $\left(\mathrm{Na}^{+}, \mathrm{Ca}^{++}, \mathrm{K}^{+} \mathrm{Cl}^{-}\right.$; Cuadro 1) muestra gran congruencia con los reportados para caballos, ya que todos los CL están cerca de 1 .

La medición de L-lactato plasmático para humanos es un procedimiento de rutina, pero en la práctica veterinaria no hace parte de la batería estándar de pruebas de laboratorio (Gómez Nieto, 2011); sin embargo el panorama es diferente en el ámbito equino, ya que este es utilizado como indicador para el pronóstico y diagnóstico en casos de cólico (Donawick et al., 1975). Además, es un indicador sensible de la presencia y la severidad del metabolismo anaerobio (TennentBrown, 2012).

Se reconoce la capacidad de PVP y TP como amortiguadores sanguíneos, y además se ha encontrado una fuerte relación entre los niveles de $\mathrm{pO}_{2}$ y la PVP durante la hipoxia en equinos a gran altura, tanto para caballos como para mulas, siendo menor la variación para estas últimas (Greene et al., 2006). También se ha encontrado un aumento en glucosa, debido a la baja de pO2 y el stress (Wickler y Greene, 2003). Así mismo, se ha observado una disminución en los valores para tCO2 durante la acidosis metabólica en caballos adultos, (Gómez Nieto, 2011).

En general, los IRs de mulas y caballos no mostraron gran diferencia, aunque se encontró algunas discrepancias con algunos reportes, e incluso variación entre los datos reportados por estos. Estas discrepancias con y entre los IRs puede estar asociada a los tres principales tipos de variaciones muéstrale: variación pre-analítica, variación analítica y variación biológica (Muñoz, 2017), variaciones que pueden alterar los resultados, dado que pueden ser técnico dependientes o derivados de la genética de los animales (Álvarez y Güell, 2011). 


\section{ConClusiones}

La comparación entre los rangos de los parámetros que permiten calcular el estado de equilibrio acido-base en caballos con los del presente estudio en mulas a gran altura indica gran similitud; de allí que los datos del estudio son idóneos para utilizarlos como referencia para mulas, tanto para otros estudios como para su uso en el diagnóstico clínico.

\section{Literatura Citada}

1. Álvarez FV, Güell MR. 2011. Interpretation of the laboratory data. FMC 18: 360-365. doi: 10.1016/S11342072(11)70141-4

2. Andersen OS, Engel K, Jorgensen K, Astrup P. 1960. A micro method for determination of $\mathrm{pH}$, carbon dioxide tension, base excess and standard bicarbonate in capillary blood. Scand J Clin Lab Inv 12: 172-176. doi: 10.3109/ 00365516009062419

3. Constable PD. 2000. Clinical assessment of acid-base status: comparison of the Henderson-Hasselbalch and strong ion approaches. Vet Clin Path 29: 115128. doi: 10.1111/j.1939-165x.2000.tb00241.x

4. Donawick WJ, Ramberg CF, Paul SR, Hiza MA. 1975. The diagnostic and prognostic value of lactate determinations in horses with acute abdominal crisis. J S Afr Vet Assoc 46: 127.

5. Friedrichs KR, Harr KE, Freeman $K P$, Szladovits B, Walton RM, Barnhart KF, Blanco-Chavez J. 2012. American Society for Veterinary Clinical Pathology. ASVCP reference interval guidelines: determination of de novo reference intervals in veterinary species and other related topics. Vet Clin Path 41: 441-453. doi: $10.1111 /$ vcp. 12006

6. Gómez Nieto DE. 2011. Comparación de dos modelos para evaluar el estado acido-base de caballos con colitis. Tesis de Maestría. Bogotá, Colombia: Univ. Nacional de Colombia. 100 p.

7. González SA. 2015. Las mulas en los llanos en el tiempo colonial y la gesta de independencia. Revista del Colegio de Médicos Veterinarios del Estado Lara 9: 44-51.

8. Greene HM, Hurson MJ, Wickler SJ. 2006. Haematological and respiratory gas changes in horses and mules exercised at altitude $(3800 \mathrm{~m})$. Equine Vet J Suppl 36: 551-556. doi: 10.1111/ j.2042-3306.2006.tb05603.x

9. Hasselbalch KA. 1917. Die berechnung der wassersroffzahl des blutes ous der freien und gebunden kohlensaure desselben, und die sauerstoffbindung des blutes als funktion der wasserstoffzahl. Biochem Z 78: 112-144.

10. Herrera BY. 2018. Vista de Perfil proteico en mulares (Equus mulus) en condiciones naturales en el departamento de Córdoba, Colombia. Rev Colomb Cienc Anim 10: 173-178. doi: 10.24188/ recia.v10.n2.2018.625

11. Ichihara K, Boyd JC, IFCC Committee on Reference Intervals and Decision Limits (C-RIDL). 2010.

An appraisal of statistical procedures used in derivation of reference intervals. Clin Chem Lab Med 48: 1537-1551. doi: 10.1515/CCLM.2010.319

12. Martínez RA, Oliver EOJ. 2016. Alcalosis metabólica hipoclorémica o alcalosis de iones fuertes: una revisión. Rev Med Vet (Bogotá) 32: 131. doi: 10.19052/mv.3862

13. McLean AK, Wang W, NavasGonzalez, FJ, Rodrigues JB. 2016. Reference intervals for hematological and blood biochemistry reference values in healthy mules and hinnies. Comp Clin Path 25: 871-878. doi: 10.1007/s00580016-2276-3

14. Mип̃oz AA. 2017. Los intervalos de referencia biológicos. Tesis de Grado. Zaragoza, España: Univ. de Zaragoza. 47 p.

15. Navarro M, Monreal L, Segura D, Armengou L, Añor S. 2005. A comparison of traditional and quantitative 
analysis of acid-base and electrolyte imbalances in horses with gastrointestinal disorders. J Vet Intern Med 19: 871-877. doi: 10.1892/0891-6640(2005)19[871:acotaq]2.0.co;2

16. Rodríguez MDA. 2015. Comparación entre el abordaje de HendersonHasselbalch y Stewart para la interpretación de la alcalosis metabólica hipoclorémica y aciduria paradójica en el modelo experimental de ovinos con pérdida de flujo abomasal. Tesis de Maestría. Bogotá, Colombia: Univ. Nacional de Colombia. $137 \mathrm{p}$.

17. Soma LR, Uboh CE, Nann L, Gerber AL. 1996. Prerace venous blood acidbase values in standardbred horses. Equine Vet J 28: 390-396. doi: 10.1111/ j.2042-3306.1996.tb03110.x

18. Stewart $P A$. 1983. Modern quantitative acid-base chemistry. Can J Physiol Pharm 61: 1444-1461. doi: 10.1139/y83-207
19. Tennent-Brown BS. 2012. Interpreting lactate measurement in critically ill horses: diagnosis, treatment, and prognosis. Compend Contin Educ Vet 34(1): E2.

20. Viu J, Jose-Cunilleras E, Armengou L, Cesarini C, Tarancón I, Rios J, Monreal L. 2010. Acid-base imbalances during a $120 \mathrm{~km}$ endurance race compared by traditional and simplified strong ion difference methods. Equine Vet J Suppl 38: 76-82. doi: 10.1111/j.20423306.2010.00213.x

21. Wickler SJ, Greene HM. 2003. The horse and high altitude. Clin Tech Equine Pract 2: 231-237. doi: 10.1053/S15347516(03)00066-0

22. Wickler, S. J., \& Greene, H. M. (2003). The horse and high altitude. Clin Tech Equine Pract 2: 231-237. https:// doi.org/10.1053/S1534-7516(03)00066-0 